\title{
Selecting Targeted Symptoms/Syndromes for Syndromic Surveillance in Rural China
}

\author{
Li Tan¹, Jie Zhang ${ }^{1}$, Liwei Cheng ${ }^{1}$, Weirong Yan ${ }^{1,2}$, Vinod K. Diwan², Lu Long1 and Shaofa \\ $\mathrm{Nie}^{\star 1}$
}

${ }^{1}$ Tongji Medical College, Wuhan City, China; ${ }^{2}$ Karolinska Institutet, Stockholm, Sweden

\section{Objective}

To select the potential targeted symptoms/syndromes as early warning indicators for epidemics or outbreaks detection in rural China.

\section{Introduction}

Patients' chief complaints (CCs) as a common data source, has been widely used in syndromic surveillance due to its timeliness, accuracy and availability (1). For automated syndromic surveillance, CCs always classified into predefined syndromic categories to facilitate subsequent data aggregation and analysis. However, in rural China, most outpatient doctors recorded the information of patients (e.g. CCs) into clinic logs manually rather than computers. Thus, more convenient surveillance method is needed in the syndromic surveillance project (ISSC). And the first and important thing is to select the targeted symptoms/syndromes.

\section{Methods}

Epidemiological analysis was conducted on data from case report system in Jingmen City (one study site in ISSC) from 2004 to 2009. Initial symptoms/syndromes were selected by literature reviews. And finally expert consultation meetings, workshops and field investigation were held to confirm the targeted symptoms/syndromes.

\section{Results}

10 kinds of infectious diseases, 6 categories of emergencies, and 4 bioterrorism events (i.e. plague, anthrax, botulism and hemorrhagic fever) were chose as specific diseases/events for monitoring (Table 1). Two surveillance schemes were developed by reviewing on 565 literatures about clinical conditions of specific diseases/events and 14 literatures about CCs based syndromic surveillance. The former one was to monitor symptoms (19 initial symptoms), and then aggregation or analysis on single or combined symptom(s); and the other one was to monitor syndromes ( 9 initial syndromes) directly (Table 2). The consultation meeting and field investigation identified three issues which should be considered: 1) the abilities of doctors especially village doctors to understand the definitions of symptoms/syndromes; 2) the workload of data collection; 3) the sensitive and specific of each symptom/syndrome. Finally, Scheme 1 was used and 10 targeted symptoms were determined (Table 2).

\section{Conclusions}

We should take the simple, stability and feasibility of operation, and also the local conditions into account before establishing a surveillance system. Symptoms were more suitable for monitoring compared to syndromes in resource-poor settings. Further evaluated and validated would be conducted during implementation. Our study might provide methods and evidences for other developing countries with limited conditions in using automated syndromic surveillance system, to construct similar early warning system.
Table 1. Epidemiological analysis on cases and emergencies data

\begin{tabular}{|c|c|c|c|c|c|}
\hline \multicolumn{2}{|l|}{ Respiratory cases } & \multicolumn{2}{|c|}{ Gastrointestinal cases } & \multicolumn{2}{|l|}{ Emergencies } \\
\hline Name & $\%$ & Name & $\%$ & Name & $\begin{array}{c}\text { Events } \\
\text { (No.) }\end{array}$ \\
\hline *Pulmonary tuberculosis & 82.38 & $\begin{array}{l}\dagger \text { Hand-foot-mouth } \\
\text { diseases }\end{array}$ & 41.73 & $\dagger \mathrm{A}(\mathrm{H} 1 \mathrm{~N} 1)$ & 10 \\
\hline$\nmid$ Mumps & 9.14 & †acillary dysentery & 28.56 & †Mumps & 5 \\
\hline †Measles & 3.35 & $\dagger$ Hepatitis A & 15.36 & $\begin{array}{l}\dagger \text { Hand-foot-mouth } \\
\text { diseases }\end{array}$ & 1 \\
\hline$\dagger$ Varicella & 2.00 & $\dagger$ Infectious diarrhea & 6.58 & $\dagger$ †acillary dysentery & 1 \\
\hline †Influenza/A(H1N1) & 1.79 & †Hepatitis E & 4.30 & Food poisoning & 2 \\
\hline$\dagger$ Rubella & 0.72 & Typhoid & 3.03 & $\begin{array}{c}\text { †Unknown reason } \\
\text { dermatitis }\end{array}$ & 1 \\
\hline Scarlet fever & 0.44 & Paratyphoid & 0.22 & & \\
\hline Pertussis & 0.15 & Amebic dysentery & 0.22 & & \\
\hline $\begin{array}{c}\text { Meningococcal } \\
\text { meningitis }\end{array}$ & 0.03 & & & & \\
\hline Total & 100.00 & Total & 100.00 & Total & 20 \\
\hline
\end{tabular}

* Chronic infectious diseases (excluded).

$\uparrow$ Selected specific diseases (top 5) or events (non-infectious excluded).

Table 2 List of symptoms/syndromes

\begin{tabular}{|c|c|c|c|c|c|}
\hline \multicolumn{4}{|c|}{ *Scheme 1} & \multicolumn{2}{|r|}{ ***Scheme 2} \\
\hline No. & Symptoms & No. & Symptoms & No. & Syndromes \\
\hline 1 & Abdominal pain & 11 & † Headache & 1 & Coma/sudden death \\
\hline 2 & Bone/muscle/joint Pain & 12 & Hematochezia & 2 & Fever \\
\hline 3 & Chills & 13 & Jaundice & 3 & Gastrointestinal \\
\hline 4 & Conjunctival hyperemia & 14 & †ucocutaneous hemorrhage & 4 & Hemorrhagic \\
\hline 5 & †Convulsion & 15 & Nasal congestion/Rhinorrhea & 5 & Influenza like illness \\
\hline 6 & Cough & 16 & $†$ Nausea/Vomitting & 6 & Neurological \\
\hline 7 & $\dagger$ Diarrhea & 17 & $\dagger$ Rach & 7 & Rash \\
\hline 8 & $\dagger$ Disturbance of consciousness & 18 & $†$ Sore throat & 8 & Respiratory \\
\hline 9 & Fatigue & 19 & Tenesmus & & \\
\hline 10 & tFever & & & & \\
\hline
\end{tabular}

* The incidence of symptom was $>=20 \%$ of specific disease(s)/event(s). $* *$ The number of times of syndromes monitored was $>=4$ times. Asthma ( 4 times) and diarrhea (5 times) were excluded due to study objectives.

$\dagger$ Final targeted symptoms.

\section{Keywords}

Syndromic surveillance; Chief complaint; Early warning

\section{Acknowledgments}

This study was funded by [European Union's] [European Atomic Energy Community's] Seventh Framework Programme ([FP7/2007-2013] [FP7/2007-2011]) under grant agreement no. [241900].

\section{References}

1.Chapman WW, Dowling JN, Wagner MM. Generating a reliable reference standard set for syndromic case classification. J Am Med Inform Assoc. 2005;12:618-29.

\section{*Shaofa Nie}

E-mail: sf_nie@mails.tjmu.edu.cn 